\title{
Knowledge, attitude and perception of tuberculosis management among tuberculosis-infected patients in resource constraint setting: field experience from Oyo state, South-West, Nigeria
}

\section{Olanrewaju Oladimeji ${ }^{1,2,3} *$, Joyce Mahlako Tsoka-Gwegweni ${ }^{1}$, Daniel Adedayo Adeyinka ${ }^{4}$, Lehlogonolo Makola ${ }^{2}$, Kabwebwe Honoré Mitonga ${ }^{3}$, Ekerette Emmanuel Udoh ${ }^{5}$, Patrick Hazangwe}

\footnotetext{
${ }^{1}$ Department of Public Health, College of Health Sciences, University of KwaZulu-Natal, South Africa

${ }^{2}$ HAST Research Programme, Humans Sciences Research Council, Durban Regional office, South Africa

${ }^{3}$ School of Public Health, Faculty of Health Sciences, University of Namibia, Oshakati, Namibia

${ }^{4}$ Department of Community Health and Epidemiology, University of Saskatchewan, Saskatoon, Canada

${ }^{5}$ Institute of Public Health, Obafemi Awolowo University, Ile-Ife, Nigeria

${ }^{6}$ Tuberculosis Unit, World Health Organization, Pretoria, Gauteng, South Africa
}

Received: 02 March 2018

Revised: 19 March 2018

Accepted: 22 March 2018

\section{*Correspondence:}

Dr. Olanrewaju Oladimeji,

E-mail: droladfb@gmail.com

Copyright: $@$ the author(s), publisher and licensee Medip Academy. This is an open-access article distributed under the terms of the Creative Commons Attribution Non-Commercial License, which permits unrestricted non-commercial use, distribution, and reproduction in any medium, provided the original work is properly cited.

\section{ABSTRACT}

Background: Inadequate knowledge and misconceptions about tuberculosis (TB) contribute to the burden of the disease by influencing patients' behavior and attitude towards the disease and undermining infection control efforts. This study assesses the level of knowledge, attitude and perception of TB and its management among TB-infected patients.

Methods: A descriptive cross-sectional was used to interview 712 TB patients using a semi-structured questionnaire between June and October 2016. Bivariate and multivariate analyses were performed with SPSS version 20.

Results: About 70\% had a good knowledge of TB. Patients' perception of stigma was high (37.5\%). A high proportion of the patients had a poor perception of their illness $(88 \%)$. Healthcare workers attitude was rated as satisfactory by $97.2 \%$ of respondents. Patients who had good knowledge about TB experienced high self-stigma $(42 \%)$ compared to those who had poor knowledge $(27.2 \%), \mathrm{p}<0.05$. Poor knowledge of TB was highest among relapse TB cases. Respondents with poor TB knowledge were less likely to be male (OR: 0.66; 95\% CI: 0.46, 0.95), married (OR: $0.24 ; 95 \%$ CI: 0.10, 0.55; p=0.001) and have tertiary education (OR: 0.06; CI: 0.37, 0.87; p=0.010). Patients with good knowledge about HIV and in the richest wealth status were $91 \%$ less likely to have high perceived stigma (OR: 0.09, 95\%CI: 0.02, 0.35; $\mathrm{p}=0.001$ ).

Conclusions: The level of knowledge of TB among patients is satisfactory, however perception of illness is poor. Tuberculosis diagnosis can create self-stigma because of the fear of being isolated and discriminated which may determine the success of treatment.

Keywords: Knowledge, Attitude, Perception, Tuberculosis, Nigeria 


\section{INTRODUCTION}

The end TB Strategy target is to accelerate the annual decline of TB incidence rates from $2 \%$ in 2015 to $10 \%$ per year by $2025 .{ }^{1}$ This strategy addresses the challenges facing many countries with high tuberculosis (TB) burden including Nigeria, which remains one of the worst TB affected countries in the world. ${ }^{2}$ Evidence suggests that the TB prevalence and mortality rates in Nigeria were previous underestimated. ${ }^{3,4}$ Despite being ranked the 4th TB affected country in the world in 2009, it was estimated that 590,000 new TB cases were reported in $2015 .{ }^{5,6}$ In order to stop the spread of TB in the country, the government of Nigeria has implemented various interventions aimed at early detection of TB and overall TB control. ${ }^{7}$ Although these initiatives contribute greatly to the management of TB, several studies carried out in Nigeria and other countries have presented TB related challenges. ${ }^{8-10}$ This includes patients' related factors such as inadequate TB knowledge as well as perceptions and attitude towards treatment. ${ }^{10-12}$ Consistently, inadequate knowledge of TB treatment especially treatment duration has been associated with treatment interruption. ${ }^{13}$ Suboptimal patients' compliance is a practical implication of poor TB knowledge. In real terms, when patients observe improvement in their health arising from the effective use of TB-medications within few weeks of treatment at the DOT centres, they stop taking medication. ${ }^{12}$ Patients whose treatment is interrupted remain infectious for longer period. They are more likely to relapse or succumb to tuberculosis as a result of treatment failure, hence foster the emergence of drug-resistant tuberculosis. ${ }^{14}$

Due to the serious negative consequences of $\mathrm{TB}$ on defaulters and its implications on the overall management of the disease, it is important to assess TB knowledge, attitude and perception among TB infected patients from a resource constraint setting in Nigeria. This information is important in providing insight on how added interventions and patient support can be provided to ensure that TB incidence and defaulting rates are reduced. With this in mind, this study sought to assess the level of knowledge, attitude and perception of TB among TB patients.

\section{METHODS}

This survey was a cross-sectional study of 712 TB patients sampled through a multistage sampling process. The first stage included the selection of local government areas (LGAs) from a sample frame of all the 16 LGAs in Ibadan and classifying the LGAs into strata with similar geographical and population characteristics before randomly selecting the LGAs. In the next stage, 25 DOT centres were randomly selected from the 16 selected LGAs. Consenting TB patients attending the DOT facility at the hospitals/health facilities were then recruited for the study. This study was conducted between June and October 2016. Data were collected with a pretested semi- structured questionnaire, which elicited information on socio-demographic characteristics of the patients, clinical profile of patients, TB knowledge and awareness, perceived TB illness and stigma, attitude of patients, health-seeking behavior and patients' satisfaction about TB services.

\section{Selection criteria}

\section{Inclusion criteria}

Inclusion criteria were patients 18 year-old or more; signed informed consent form after due explanations of the study purpose; participants currently on TB treatment; participant with all forms of $\mathrm{TB}$ including extra pulmonary TB (EP TB).

\section{Exclusion criteria}

Exclusion criteria were age less than 18 years old; unable to provide informed consent (e.g. unfamiliarity with language of patient information/consent forms mentally impaired).

\section{Data management and analysis}

The overall TB knowledge was assessed by summing TB knowledge related questions into a single knowledge variable. The questions about knowledge included: (1) availability of cure for TB, knowledge about vaccination for $\mathrm{TB}$, if vaccination can protect against $\mathrm{TB}$, knowledge of mode of TB transmission (through air when coughing or sneezing), knowledge of other preventions (covering mouth and nose when coughing or sneezing, good nutrition, but not by praying, closing windows at home, and to avoid shaking hands), ability to enumerate more than four of the following signs and symptoms for TB cough that lasts longer than 3 weeks, coughing up blood, weight loss, fever, chest pain, shortness of breath..$^{2-6} \mathrm{~A}$ score of one (1) was given to correct responses and zero (0) for incorrect/do not know responses. Responses were added together for each respondent to generate a knowledge score ranging from a minimum of 0 to maximum of 6 . Knowledge variables were then dichotomized as poor (score: 0-3) and good (score: 4-6). Similarly, patients' perceived stigma variable was computed using 10 questions that asked about the patients' thoughts or feelings about their social experiences or the reactions of others from having TB. The patients whose response was 'Yes' to a negative social experience or reaction were scored ' 1 ' and those who said ' $N o^{\prime}$ ' were scored ' 0 ', with a total score of 10 points. Overall, perceived stigma was dichotomized as low stigma (Score: 0-5) and high stigma (score: 6-10). Illness perception of patients was evaluated using a 9item illness perception of 5-point Likert scale. TB service was assessed by asking the patients about the quality of TB services offered, satisfaction with the environment, and health care workers attitude. 
Data were analyzed with the statistical package IBM SPSS 20. Univariate analysis was conducted to describe all variables, while bivariate analyses were performed with Chi-Square test. The dependent variables were knowledge, perceived stigma and quality of service perception. A multivariate analysis employed backward step-wise model of binary logistic regression to identify predictors of the dependent variables. Statistical significance for association was set at $\mathrm{p}<0.05$.

\section{RESULTS}

The mean age of respondent was 37.8 (SD: 13.7) years. Majority of respondents in the study were female $(61.7 \%)$. The socio-demographic characteristics of respondents are presented in Table 1. Majority of the respondents in the study were either married or cohabiting, followed by those who were never married. Respondents who had secondary education as their highest level of education predominated the study $(40.7 \%)$, followed by those who had primary education $(25.4 \%)$ and tertiary education $(25.4 \%)$. The respondents of Yoruba tribe predominated the study with $94.4 \%$. The majority were in the poorer $(47.5 \%)$ and middle wealth categories.

Table 2 presents the clinical profile of the patients. Out of the patients in the study, $7.4 \%$ were reactive to HIV. Majority of the patients $(85.9 \%)$ were new TB cases on treatment, $7.4 \%$ were retreatment cases, while $6.7 \%$ were relapsed cases. Most $(89.1 \%)$ of the patents were diagnosed of TB through microscopy, 3.7\% through GeneXpert, 5.2\% through Culture and 2\% through molecular Line probe assay. More than $50 \%$ of the patients had been on treatment for 6-8 months, while $44.8 \%$ were on treatment for about $\leq 5$ months, and $3 \%$ had been on treatment for more $\geq 9$ months.

Table 3 presents information about knowledge of TB among the patients. Most respondents reported that they first learnt about TB from a health worker (32.2\%). Other sources of information were radio $(24.4 \%)$ and family, friend neighbor or colleagues $(12.6 \%)$. More than two third of respondents reported that the information about TB was fully understandable $(67.2 \%)$, and $26 \%$ said the information was partly understandable, while $6 \%$ could not understand the information. Of the respondents who said that the information was partly or not understandable, $31.5 \%$ felt that the information was too much and $28.6 \%$ said the information was not clear. Only about half $(51.4 \%)$ reported that the information they received about TB was adequate. About $28.5 \%$ of the respondents perceived that $\mathrm{TB}$ infection is a common disease in their community. About $89 \%$ of the respondents said that $\mathrm{TB}$ diseases could be cured while the remaining proportion did not know if it could be cured. In addition, only about $60 \%$ believed that the TB vaccine could protect against TB. More than a third of patients $(35 \%)$ did not have good knowledge of the mode of transmission of TB; $16.3 \%$ had poor knowledge of ways to prevent the transmission of TB. Knowledge of signs and symptoms of TB was good among $68.3 \%$ of the respondents.

Majority of respondents (88\%) reported that treatment for TB patients is accessible at government hospital. About $87.2 \%$ of the respondents said that 6 months was the duration of treatment for $\mathrm{TB}$, while $2 \%$ reported 9 months duration for TB treatment. Overall, the percentage of respondents who had good knowledge about TB was approximately $70 \%$. More male $(74.6 \%)$ than female $(66.5 \%)$ had significantly good knowledge about TB.

Few respondents had misconception about TB (Table 4). Some patients felt that TB could be transmitted by sharing dishes (13.9\%), eating from the same plate $(13.5 \%)$, and by touching items in public places. The misconceptions about preventions were; avoiding sharing dishes $(19.5 \%)$ and hand washing after touching items in public places $(36.5 \%)$.

Figures 1 and 2 present the stigma attitude among TB patients. It was observed that more than a quarter of the respondents $(37.5 \%)$ experienced high stigma against $62.5 \%$ who experienced low stigma. About $59.7 \%$ of respondents felt ashamed of having TB; $72.9 \%$ were of the opinion that people who have HIV should be concerned about TB. Most of the respondents felt that TB could affect their social relations (50.7\%), their work $(54.2 \%)$ and even their marital relations (36\%). Also, $32.4 \%$ felt that TB could affect their chances of getting married. Some of the patients however preferred to be isolated (18.4\%). A high proportion (38.6\%) felt that TB affects their family responsibilities. Most of the patients $(88 \%)$ had a poor perception of their illness.

Table 5 presents the health-seeking behavior of the respondents. More than half $(64.6 \%)$ reported that they usually seek care at a government clinic or hospital when ill, while $28.7 \%$ said they sought care at private clinics. Majority of the respondents $(42.1 \%)$ reported that they generally seek health care at clinic or hospital monthly. Majority of the respondent rated the services offered at the TB Centre's as excellent (60.4\%), very good (29.4\%) and good $(8.7 \%)$, only $1.5 \%$ rated the services as bad. Similarly, majority of the respondents rated the attitude of TB health care workers as positive (97.2\%). More than a third $(36.1 \%)$ reported that there were usually too many people taking treatment at the TB centres.

\section{Bivariate and multivariate results}

Table 6 shows results of association between independent factors and the dependent factors TB knowledge indicator and patient's self-stigma level. Age did not show any significant association with both TB knowledge and patient's stigma level. Educational status of patients was associated with TB knowledge, as poor knowledge about 
TB was higher among those who had no formal education. Patient's whose home was more than $10 \mathrm{~km}$ away from a health facility had a greater proportion of patients who had poor TB knowledge compared to patients who lived closer to a health facility. Patients who were in the poorest wealth quintile had a higher tendency to experience high stigma compared to those who were either in the rich and richest wealth categories.

Table 1: Socio-demographic characteristic of the respondents stratified by sex.

\begin{tabular}{|c|c|c|c|c|c|}
\hline & $\begin{array}{l}\text { Frequency } \\
(\mathrm{n}=712)\end{array}$ & Percentage (\%) & Male & Female & P value \\
\hline Sex & & & 38.3 & 61.7 & \\
\hline \multicolumn{6}{|l|}{ Age group } \\
\hline $18-24$ & 107 & 15.6 & 15.9 & 15.8 & \multirow{4}{*}{0.109} \\
\hline $25-34$ & 194 & 28.5 & 28.7 & 28.1 & \\
\hline $40-44$ & 190 & 27.9 & 23.4 & 30.9 & \\
\hline$\geq 45$ & 190 & 27.9 & 32.1 & 25.3 & \\
\hline \multicolumn{6}{|l|}{ Marital status } \\
\hline Never married & 185 & 26.0 & 25.7 & 26.0 & \multirow{3}{*}{0.993} \\
\hline Married/co-habiting & 495 & 69.5 & 69.9 & 69.5 & \\
\hline Separated/widowed/widower & 32 & 4.5 & 4.4 & 4.6 & \\
\hline \multicolumn{6}{|l|}{ Family type } \\
\hline a) Monogamous & 468 & 65.7 & & & \multirow{3}{*}{0.630} \\
\hline b) Polygamous & 244 & 34.3 & & & \\
\hline Total & 712 & 100.0 & & & \\
\hline \multicolumn{6}{|l|}{ Educational status } \\
\hline No formal education & 60 & 8.4 & 9.6 & 7.7 & \multirow{4}{*}{0.776} \\
\hline Primary & 181 & 25.4 & 24.3 & 26.2 & \\
\hline Secondary & 290 & 40.7 & 41.5 & 40.1 & \\
\hline Tertiary & 181 & 25.4 & 24.6 & 26.0 & \\
\hline \multicolumn{6}{|l|}{ Occupation } \\
\hline a) Unemployed & 50 & 7.0 & 9.9 & 5.2 & \multirow{7}{*}{0.039} \\
\hline b) Student & 102 & 14.3 & 13.6 & 14.8 & \\
\hline c) Trading & 282 & 39.6 & 34.9 & 42.4 & \\
\hline d) Farming & 32 & 4.5 & 5.1 & 4.1 & \\
\hline e) Self-employed & 177 & 24.9 & 23.9 & 25.5 & \\
\hline f) Civil servant & 42 & 5.9 & 8.5 & 4.3 & \\
\hline $\begin{array}{l}\text { g) Employed with private } \\
\text { organization }\end{array}$ & 27 & 3.8 & 4.0 & 3.6 & \\
\hline \multicolumn{6}{|l|}{ Religious } \\
\hline a) Christian & 339 & 47.6 & 49.3 & 46.5 & \multirow{3}{*}{0.762} \\
\hline b) Islam & 370 & 52.0 & 50.4 & 53.1 & \\
\hline c) Traditional worshiper & 3 & 0.4 & 0.4 & 0.5 & \\
\hline \multicolumn{6}{|l|}{ Ethnic group } \\
\hline a) Yoruba & 672 & 94.4 & 91.5 & 96.1 & \multirow{3}{*}{0.014} \\
\hline b) Igbo & 26 & 3.7 & 6.2 & 2.1 & \\
\hline c) Hausa & 14 & 2.0 & 2.2 & 1.8 & \\
\hline \multicolumn{6}{|l|}{ Distance from home to facility } \\
\hline a) $<5 \mathrm{~km}$ & 261 & 36.7 & 37.9 & 36.0 & \multirow{3}{*}{0.288} \\
\hline b) $5 \mathrm{~km}-10 \mathrm{~km}$ & 262 & 36.8 & 39.0 & 35.5 & \\
\hline c) $>10 \mathrm{~km}$ & 189 & 26.5 & 23.2 & 28.5 & \\
\hline \multicolumn{6}{|l|}{ Wealth quintile } \\
\hline Poorest & 58 & 8.1 & 8.5 & 8.0 & \multirow{5}{*}{0.710} \\
\hline Poorer & 338 & 47.5 & 47.1 & 47.8 & \\
\hline Middle & 232 & 32.6 & 32.4 & 32.6 & \\
\hline Richer & 63 & 8.8 & 8.1 & 9.3 & \\
\hline Richest & 21 & 2.9 & 4.0 & 2.3 & \\
\hline
\end{tabular}


Table 2: Clinical profile of the respondents.

\begin{tabular}{|c|c|c|c|c|c|}
\hline & $\begin{array}{l}\text { Frequency } \\
(n=712)\end{array}$ & Percentage (\%) & Male & Female & P value \\
\hline \multicolumn{6}{|l|}{ HIV serostatus } \\
\hline Reactive & 50 & 7.4 & 8.6 & 6.7 & \multirow{3}{*}{0.650} \\
\hline Non-reactive & 582 & 86.2 & 85.6 & 86.8 & \\
\hline Don’t know & 43 & 6.4 & 5.8 & 6.5 & \\
\hline \multicolumn{6}{|c|}{ Treatment classification } \\
\hline Retreatment & 49 & 7.4 & 7.9 & 6.9 & \multirow{3}{*}{0.863} \\
\hline Relapse & 44 & 6.7 & 6.3 & 6.9 & \\
\hline New treatment & 566 & 85.9 & 85.8 & 86.2 & \\
\hline \multicolumn{6}{|c|}{ Method of diagnosis } \\
\hline Microscopy & 583 & 89.1 & 89.1 & 89.1 & \multirow{4}{*}{0.720} \\
\hline Genexpert & 24 & 3.7 & 2.8 & 4.2 & \\
\hline Culture & 34 & 5.2 & 6.0 & 4.7 & \\
\hline LPA & 13 & 2.0 & 2.0 & 2.0 & \\
\hline \multicolumn{6}{|c|}{ Duration of treatment } \\
\hline $0-5$ months & 296 & 44.8 & 43. & 45.6 & \multirow{3}{*}{0.183} \\
\hline 6-8 months & 343 & 52.0 & 51.8 & 52.2 & \\
\hline$>9$ months & 21 & 3.2 & 4.8 & 2.2 & \\
\hline
\end{tabular}

Table 3: Patients knowledge about TB.

\begin{tabular}{|lllll|}
\hline Where respondent first learnt about TB & Frequency & Percentage (\%) & Male & Female \\
\hline Pewspapers and magazines & & & & \\
\hline Radio & 44 & 6.2 & 5.5 & 6.4 \\
\hline TV & 174 & 24.4 & 25.0 & 24.1 \\
\hline Brochures, posters and other printed materials & 46 & 6.5 & 7.4 & 5.9 \\
\hline Health workers & 60 & 8.4 & 8.1 & 8.7 \\
\hline Family, friends, neighbors and colleagues & 229 & 32.2 & 31.6 & 34.6 \\
\hline Religious leaders & 90 & 12.6 & 14.3 & 11.6 \\
\hline Teachers & 9 & 1.3 & 0.4 & 1.8 \\
\hline Others & 20 & 2.8 & 3.3 & 2.5 \\
\hline Information got about TB was understandable & 40 & 5.6 & 4.4 & 4.3 \\
\hline Yes fully & 460 & 67.2 & & \\
\hline Yes partly & 178 & 26.0 & 66.0 & 68.0 \\
\hline No & 47 & 6.9 & 26.0 & 25.8 \\
\hline Why information about TB was partly or not understandable & & 8.0 & 6.2 \\
\hline The information language used was difficult & 37 & 15.4 & & \\
\hline The information is not clear & 69 & 28.6 & 23.9 & 15.5 \\
\hline Too much information & 76 & 31.5 & 27.2 & 31.1 \\
\hline Information was incorrect & 9 & 3.7 & 5.4 & 2.7 \\
\hline Information not complete & 50 & 20.7 & 28.3 & 16.2 \\
\hline Adequacy of information got about TB & & & & \\
\hline Adequate & 366 & 51.4 & 51.5 & 51.5 \\
\hline Not adequate & 272 & 38.2 & 40.8 & 36.7 \\
\hline Don't know & 74 & 10.4 & 7.7 & 11.8 \\
\hline TB a common disease in the community & & & \\
\hline Yes & 203 & 28.5 & 34.2 & 25.1 \\
\hline No & 395 & 55.5 & 50.0 & 59.0 \\
\hline Don't know & 114 & 16.0 & 15.8 & 15.9 \\
\hline Total & 712 & 100.0 & & \\
\hline
\end{tabular}




\begin{tabular}{|c|c|c|c|c|c|}
\hline & Frequency & Percentage (\%) & Male & Female & P value \\
\hline \multicolumn{6}{|l|}{ TB can be cured } \\
\hline Yes & 631 & 88.6 & 88.6 & 88.6 & \multirow{4}{*}{0.408} \\
\hline No & 42 & 5.9 & 7.0 & 5.2 & \\
\hline Don't know & 39 & 5.5 & 4.4 & 6.2 & \\
\hline Total & 712 & 100.0 & & & \\
\hline \multicolumn{6}{|l|}{ There is vaccination against $\mathrm{TB}$} \\
\hline Yes & 424 & 59.6 & 63.6 & 57.2 & \multirow{4}{*}{0.180} \\
\hline No & 174 & 24.4 & 23.2 & 25.3 & \\
\hline Don't know & 114 & 16.0 & 13.2 & 17.5 & \\
\hline Total & 712 & 100.0 & & & \\
\hline \multicolumn{6}{|l|}{ TB vaccination protects against $\mathrm{TB}$} \\
\hline Yes & 349 & 49.0 & 51.8 & 47.4 & \multirow{4}{*}{0.373} \\
\hline No & 186 & 26.1 & 26.1 & 26.2 & \\
\hline Don't know & 177 & 24.9 & 22.1 & 26.4 & \\
\hline Total & 712 & 100.0 & & & \\
\hline \multicolumn{6}{|l|}{ Knowledge of mode of transmission } \\
\hline Do not have good knowledge of transmission & 249 & 35.0 & & & \\
\hline Have good knowledge of transmission & 463 & 65.0 & & & \\
\hline \multicolumn{6}{|l|}{ Knowledge of transmission prevention } \\
\hline Poor & 116 & 16.3 & 15.4 & 16.6 & \multirow{2}{*}{0.676} \\
\hline Good & 596 & 83.7 & 84.6 & 83.4 & \\
\hline \multicolumn{6}{|l|}{ Knowledge of Signs and symptoms of TB } \\
\hline Poor knowledge of symptoms & 226 & 31.7 & 28.7 & 33.5 & \multirow{2}{*}{0.180} \\
\hline Good knowledge of symptoms & 486 & 68.3 & 71.3 & 66.5 & \\
\hline \multicolumn{6}{|l|}{ Any family member has TB } \\
\hline Yes & 81 & 11.4 & 13.2 & 10.3 & \multirow{3}{*}{0.207} \\
\hline No & 555 & 77.9 & 78.3 & 77.9 & \\
\hline Don't know & 76 & 10.7 & 8.5 & 11.8 & \\
\hline \multicolumn{6}{|l|}{ Any neighbors has TB } \\
\hline Yes & 97 & 13.6 & 14.7 & 13.0 & \multirow{3}{*}{0.660} \\
\hline No & 465 & 65.3 & 65.8 & 65.1 & \\
\hline Don't know & 150 & 21.1 & 19.5 & 21.9 & \\
\hline \multicolumn{6}{|l|}{ Any friend has TB } \\
\hline Yes & 96 & 13.5 & 14.0 & 13.2 & \multirow{3}{*}{0.778} \\
\hline No & 475 & 66.7 & 67.6 & 66.3 & \\
\hline Don't know & 141 & 19.8 & 18.4 & 20.5 & \\
\hline \multicolumn{6}{|l|}{ Knowledge of seriousness of TB disease } \\
\hline Very serious & 563 & 79.1 & 79.8 & 78.8 & \multirow{4}{*}{0.450} \\
\hline Somewhat serious & 60 & 8.4 & 7.0 & 9.1 & \\
\hline Not very serious & 49 & 6.9 & 6.2 & 7.3 & \\
\hline I have no idea & 40 & 5.6 & 7.0 & 4.8 & \\
\hline \multicolumn{6}{|c|}{ Knowledge of Seriousness of problem of TB in the county or region } \\
\hline Very serious & 403 & 56.6 & 59.6 & 54.9 & \\
\hline Somewhat serious & 137 & 19.2 & 19.5 & 19.1 & \\
\hline Not very serious & 61 & 8.6 & 7.4 & 9.1 & 0.492 \\
\hline I have no idea & 111 & 15.6 & 13.6 & 16.9 & \\
\hline Knowledge of number of types of TB & & & & & \\
\hline One type & 247 & 36.0 & 34.0 & 37.3 & \\
\hline Two types & 270 & 39.3 & 40.4 & 38.7 & \\
\hline More than two & 170 & 24.7 & 25.7 & 24.0 & 0.671 \\
\hline Total & 687 & 100.0 & & & \\
\hline System & 25 & & & & \\
\hline
\end{tabular}




\begin{tabular}{|lllll|}
\hline & Frequency & Percentage (\%) & Male & Female \\
\hline Phere can TB patient get treatment & & & & \\
\hline Governmental hospital & 629 & 88.3 & 88.6 & 88.2 \\
\hline Private hospitals & 10 & 1.4 & 1.8 & 1.1 \\
\hline Non-government organization clinic & 20 & 2.8 & 2.9 & 2.7 \\
\hline Don't know & 53 & 7.4 & 6.6 & 8.0 \\
\hline Reaction when diagnosed of TB & & & & \\
\hline Fear & 379 & 55.7 & 53.8 & 56.8 \\
\hline Surprise & 173 & 25.4 & 26.5 & 24.7 \\
\hline Shame & 24 & 3.5 & 3.1 & 3.8 \\
\hline Embarrassment & 45 & 6.6 & 8.5 & 5.5 \\
\hline Sadness/hopelessness & 60 & 8.8 & 8.1 & 9.3 \\
\hline Who did you talk to about your illness & & & & \\
\hline Doctor or other medical workers & 289 & 42.6 & 39.8 & 44.1 \\
\hline Spouse & 125 & 18.4 & 18.4 & 18.5 \\
\hline Parent & 129 & 19.0 & 21.1 & 17.7 \\
\hline Children & 24 & 3.5 & 3.8 & 3.4 \\
\hline Other family members & 36 & 5.3 & 5.4 & 5.3 \\
\hline Close friend & 68 & 10.0 & 11.1 & 9.4 \\
\hline No one & 8 & 1.2 & 0.4 & 1.7 \\
\hline Total & 679 & 100.0 & & \\
\hline Knowledge of duration of treatment of TB & & & & \\
\hline Less than 3 months & 20 & 2.9 & 3.0 & 2.8 \\
\hline 3 months & 24 & 3.5 & 2.7 & 3.8 \\
\hline 6 months & 601 & 87.2 & 89.0 & 86.3 \\
\hline 9 months & 11 & 1.6 & 1.1 & 1.9 \\
\hline Year & 11 & 1.6 & 1.9 & 1.4 \\
\hline Don't know & 22 & 3.2 & 2.3 & 3.8 \\
\hline TB knowledge indicator & & & & \\
\hline Good knowledge & 495 & 69.5 & 74.6 & 66.5 \\
\hline Poor knowledge & 217 & 30.5 & 25.4 & 33.5 \\
\hline
\end{tabular}

Table 4: Respondent misconception about TB.

\begin{tabular}{|lll|}
\hline & Frequency & Percentage (\%) \\
\hline TB transmitted through handshake & 47 & 6.6 \\
\hline TB transmitted by sharing dishes & 99 & 13.9 \\
\hline TB transmitted through eating from the same plate & 96 & 13.5 \\
\hline TB transmitted through touching items in public & 78 & 11.0 \\
\hline Prevented by avoiding to shake hands & 48 & 6.7 \\
\hline Prevented by avoiding sharing dishes & 139 & 19.5 \\
\hline Washing hands after touching items in public places & 260 & 36.5 \\
\hline Prevented by praying & 65 & 9.1 \\
\hline TB can be cured by herbal remedies & 16 & 2.2 \\
\hline TB can be cured by praying & 60 & 8.4 \\
\hline
\end{tabular}

Knowledge of TB was significantly associated with selfstigma attitude of patients, as patients who had good knowledge about TB experienced high self-stigma (42\%) compared to those who had poor knowledge (27.2\%). The result showed that TB quality of service as perceived by the patients was associated with knowledge of TB and patient's self-stigma (Table 6).

Table 7 showed that there was an association between the treatment classifications of patients with TB knowledge.
Among the patients with poor knowledge, the relapse cases were significantly higher $(52.3 \%)$ than those who were on either new treatment $(28.6 \%)$ or retreatment $(36.7 \%)$. Table 8 shows that knowledge and stigma attitude was related with perception of quality of service.

Table 9 shows the predictors of poor knowledge of TB among patients. There was a significantly lower odds of having poor TB knowledge among males (OR: 0.66; $95 \%$ CI: $0.46,0.95 ; \mathrm{p}=0.027$ ), married/cohabiting respondents 
(OR: 0.24; CI: 0.10, 0.55; $\mathrm{p}=0.001)$ and either separated and or patients who lost a spouse (OR: 0.30; CI: 0.14, $0.66 ; \mathrm{p}=0.003)$ compared to those who were never married. Patients who had tertiary education as their highest level of education were significantly less likely to have poor TB knowledge. With respect to self-stigma of the patients, only wealth quintile remained significant in predicting high self-stigma of the patients (Table 10). Patients in the richest wealth category had a much lesser odds of experiencing high self-stigma compared to respondents in the poorest wealth category (OR: 0.091, CI: $0.02,0.35 ; \mathrm{p}=0.001$ ).

Table 5: Health seeking behavior and TB service assessment.

\begin{tabular}{|c|c|c|}
\hline & Frequency & Valid percentage $(\%)$ \\
\hline \multicolumn{3}{|l|}{ Where do you usually go if you are sick } \\
\hline Private clinic & 204 & 28.7 \\
\hline Government clinic or hospital & 460 & 64.6 \\
\hline Traditional healer & 41 & 5.8 \\
\hline Clinic run by non-governmental organization & 7 & 1.0 \\
\hline Total & 712 & 100.0 \\
\hline \multicolumn{3}{|c|}{ How often do you generally seek health care at a clinic or hospital } \\
\hline Monthly or more & 300 & 42.1 \\
\hline Four times a year or more & 134 & 18.8 \\
\hline Twice a year or more & 109 & 15.3 \\
\hline Once per year & 84 & 11.8 \\
\hline Less than once a year but at least twice in past 5 years & 22 & 3.1 \\
\hline Once in past 5 years & 26 & 3.7 \\
\hline Never in past 5 years & 37 & 5.2 \\
\hline Total & 712 & 100.0 \\
\hline \multicolumn{3}{|l|}{ What do you think about TB center services offered } \\
\hline Excellent & 430 & 60.4 \\
\hline Very good & 209 & 29.4 \\
\hline Good & 62 & 8.7 \\
\hline $\mathrm{Bad}$ & 11 & 1.5 \\
\hline Total & 712 & 100.0 \\
\hline \multicolumn{3}{|c|}{ What do you think about TB health care workers attitude } \\
\hline Positive & 692 & 97.2 \\
\hline Negative & 20 & 2.8 \\
\hline Total & 712 & 100.0 \\
\hline \multicolumn{3}{|l|}{ What do you think about TB center appearance } \\
\hline Excellent & 326 & 45.8 \\
\hline Very good & 237 & 33.3 \\
\hline Good & 138 & 19.4 \\
\hline $\mathrm{Bad}$ & 11 & 1.5 \\
\hline Total & 712 & 100.0 \\
\hline \multicolumn{3}{|c|}{ What do you think about number of people seeking treatment in TB center } \\
\hline Too many & 257 & 36.1 \\
\hline Many & 286 & 40.2 \\
\hline Few & 159 & 22.3 \\
\hline Very few & 10 & 1.4 \\
\hline Total & 712 & 100.0 \\
\hline \multicolumn{3}{|l|}{ What do you think about waiting time in TB center } \\
\hline 15 minutes & 312 & 45.9 \\
\hline $15-30$ minutes & 247 & 36.4 \\
\hline 30 minutes -1 hour & 106 & 15.6 \\
\hline More than 2 hours & 14 & 2.1 \\
\hline Total & 679 & 100.0 \\
\hline \multicolumn{3}{|l|}{ TB service assessments } \\
\hline Poor service & 181 & 25.4 \\
\hline Good service & 531 & 74.6 \\
\hline
\end{tabular}


Table 6: Association between socio-demographic factors and TB knowledge and patients' perceived stigma level.

\begin{tabular}{|c|c|c|c|c|c|c|}
\hline & \multicolumn{3}{|c|}{ General knowledge about TB } & \multicolumn{3}{|c|}{ Patients perceived-stigma level } \\
\hline & Good & Poor & Chi-square (df) & Low stigma & High stigma & $\mathrm{P}$ value \\
\hline \multicolumn{7}{|l|}{ Sex } \\
\hline Male & 41.0 & 59.0 & $\mathrm{p}=0.022$ & 37.2 & 62.8 & 0.439 \\
\hline Female & 31.9 & 68.1 & & 40.1 & 59.9 & \\
\hline \multicolumn{7}{|l|}{ Age group } \\
\hline $18-24$ & 74.0 & 26.0 & $\mathrm{P}=0.329$ & 65.2 & 34.8 & 0.962 \\
\hline $25-34$ & 71.2 & 28.8 & & 63.4 & 36.6 & \\
\hline $35-44$ & 69.5 & 30.5 & & 62.3 & 37.7 & \\
\hline$\geq 45$ & 66.8 & 33.2 & & 61.1 & 38.9 & \\
\hline \multicolumn{7}{|l|}{ Total } \\
\hline \multicolumn{7}{|l|}{ Marital status } \\
\hline Never married & 71.9 & 28.1 & $\mathrm{P}=0.001$ & 62.7 & 37.3 & 0.998 \\
\hline Married/co-habiting & 70.5 & 29.5 & & 62.4 & 37.6 & \\
\hline Separated/widowed/widower & 40.6 & 59.4 & & 62.5 & 37.5 & \\
\hline \multicolumn{7}{|l|}{ Total } \\
\hline \multicolumn{7}{|l|}{ Family type } \\
\hline a) Monogamous & 65.4 & 34.6 & $\mathrm{P}=0.001$ & 63.7 & 36.3 & 0.370 \\
\hline b) Polygamous & 77.5 & 22.5 & & 60.2 & 39.8 & \\
\hline \multicolumn{7}{|l|}{ Total } \\
\hline \multicolumn{7}{|l|}{ Educational status } \\
\hline No formal education & 63.3 & 36.7 & $\mathrm{P}=0.023$ & 56.7 & 43.3 & 0.201 \\
\hline Primary & 66.3 & 33.7 & & 57.5 & 42.5 & \\
\hline Secondary & 75.9 & 24.1 & & 64.1 & 35.9 & \\
\hline Tertiary & 64.6 & 35.4 & & 66.9 & 33.1 & \\
\hline \multicolumn{7}{|l|}{ Total } \\
\hline \multicolumn{7}{|l|}{ Occupation } \\
\hline a) Unemployed & 72.0 & 28.0 & $\mathrm{P}=0.739$ & 46.0 & 54.0 & 0.175 \\
\hline b) Student & 74.5 & 25.5 & & 62.7 & 37.3 & \\
\hline c) Trading & 68.4 & 31.6 & & 63.1 & 36.9 & \\
\hline d) Farming & 71.9 & 28.1 & & 62.5 & 37.5 & \\
\hline e) Self-employed & 65.5 & 34.5 & & 64.4 & 35.6 & \\
\hline f) Civil servant & 73.8 & 26.2 & & 73.8 & 26.2 & \\
\hline g) Employed with private organization & 74.1 & 25.9 & & 55.6 & 44.4 & \\
\hline \multicolumn{7}{|l|}{ Total } \\
\hline \multicolumn{7}{|l|}{ Religious } \\
\hline a) Christian & 69.0 & 31.0 & $\mathrm{P}=0.506$ & 59.3 & 40.7 & 0.112 \\
\hline b) Islam & 69.7 & 30.3 & & 65.1 & 34.9 & \\
\hline c) Traditional worshiper & 100.0 & & & 100.0 & & \\
\hline \multicolumn{7}{|l|}{ Total } \\
\hline \multicolumn{7}{|l|}{ Distance from home to facility } \\
\hline a) $<5 \mathrm{~km}$ & 70.5 & 29.5 & $\mathrm{P}=0.001$ & 62.1 & 37.9 & 0.535 \\
\hline b) $5 \mathrm{~km}-10 \mathrm{~km}$ & 75.6 & 24.4 & & 64.9 & 35.1 & \\
\hline c) $>10 \mathrm{~km}$ & 59.8 & 40.2 & & 59.8 & 40.2 & \\
\hline \multicolumn{7}{|l|}{ Total } \\
\hline \multicolumn{7}{|l|}{ Wealth quintile } \\
\hline Poorest & 72.4 & 27.6 & $\mathrm{P}=0.424$ & 36.2 & 63.8 & $<0.001$ \\
\hline Poorer & 67.2 & 32.8 & & 63.9 & 36.1 & \\
\hline Middle & 70.7 & 29.3 & & 66.8 & 33.2 & \\
\hline Richer & 69.8 & 30.2 & & 57.1 & 42.9 & \\
\hline Richest & 85.7 & 14.3 & & 81.0 & 19.0 & \\
\hline Total & & & & & & \\
\hline TB knowledge indicator & & & & & & \\
\hline Good knowledge & & & & 58.0 & 42.0 & $<0.001$ \\
\hline Poor knowledge & & & & 72.8 & 27.2 & \\
\hline
\end{tabular}


Table 7: Association between patients' clinical profile and TB knowledge.

\begin{tabular}{|c|c|c|c|}
\hline & \multicolumn{3}{|c|}{ TB knowledge } \\
\hline & Good & Poor & $P$ value \\
\hline \multicolumn{4}{|l|}{ HIV serostatus } \\
\hline Reactive & 76.0 & 24.0 & 0.267 \\
\hline Non-reactive & 69.4 & 30.6 & \\
\hline Don't know & 60.5 & 39.5 & \\
\hline \multicolumn{4}{|c|}{ Treatment classification } \\
\hline Retreatment & 63.3 & 36.7 & 0003 \\
\hline Relapse & 47.7 & 52.3 & \\
\hline New treatment & 71.4 & 28.6 & \\
\hline \multicolumn{4}{|c|}{ Method of diagnosis } \\
\hline Microscopy & 69.8 & 30.2 & 0.009 \\
\hline Genexpert & 50.0 & 50.0 & \\
\hline Culture & 85.3 & 14.7 & \\
\hline LPA & 46.2 & 53.8 & \\
\hline \multicolumn{4}{|c|}{ Duration of treatment } \\
\hline $0-5$ months & 66.9 & 33.1 & 0.282 \\
\hline 6-8 months & 72.0 & 28.0 & \\
\hline$>9$ months & 61.9 & 38.1 & \\
\hline
\end{tabular}

Table 8: Association between TB knowledge and patients' perceived stigma level with TB quality of service perception.

\begin{tabular}{|lccc|}
\hline & \multicolumn{2}{l}{ TB quality of service perception } & \\
\cline { 2 - 3 } & Poor perception & Good perception & P value \\
\hline TB knowledge indicator & & & \\
\hline Good & 28.3 & 71.7 & 0.008 \\
\hline Poor & 18.9 & 81.1 & \\
\hline Patient's perceived-stigma level & & & 0.020 \\
\hline Low stigma & 22.5 & 77.5 & \\
\hline High stigma & 30.3 & 69.7 & \\
\hline
\end{tabular}

Table 9: Predictors of poor knowledge of TB.

\begin{tabular}{|c|c|c|c|}
\hline & AOR & $\mathbf{9 0 \%}$ confidence interval & $P$ value \\
\hline Male sex & 0.66 & $0.46,0.95$ & 0.027 \\
\hline \multicolumn{4}{|l|}{ Marital status } \\
\hline Never married & 1 & & 0.004 \\
\hline Married/co-habiting & 0.244 & $0.10,0.55$ & 0.001 \\
\hline Seperated/widowed & 0.306 & $0.14,0.66$ & 0.003 \\
\hline \multicolumn{4}{|l|}{ Family type } \\
\hline Monogamous family type & 1.80 & $1.23,2.64$ & 0.002 \\
\hline \multicolumn{4}{|l|}{ Educational status } \\
\hline No formal education & 1 & & 0.050 \\
\hline Primary & 1.01 & $0.52,1.96$ & 0.969 \\
\hline Secondary & 0.84 & $0.52,1.37$ & 0.504 \\
\hline Tertiary & 0.56 & $0.37,0.87$ & 0.010 \\
\hline \multicolumn{4}{|c|}{ Distance from home to facility } \\
\hline a) $<5 \mathrm{~km}$ & 1 & & 0.008 \\
\hline b) $5 \mathrm{~km}-10 \mathrm{~km}$ & 0.67 & $0.43,1.02$ & 0.064 \\
\hline c) $>10 \mathrm{~km}$ & 0.53 & $0.34,0.81$ & 0.002 \\
\hline
\end{tabular}

Chi-s $=46.332, \mathrm{df}=12, \mathrm{p}=0.000$, Nagelkerke R-square $=0.093$; Note: Factors in the equation include sex, age group, marital status, family type, educational status, occupation and distance from facility. 
Table 10: Predictors for high perceived-stigma.

\begin{tabular}{|c|c|c|c|}
\hline & AOR & $90 \%$ confidence interval & P value \\
\hline \multicolumn{4}{|l|}{ Wealth quintile } \\
\hline Poorest & 1 & & 0.000 \\
\hline Poorer & 0.308 & $0.16,0.56$ & 0.000 \\
\hline Middle & 0.267 & $0.14,0.50$ & 0.000 \\
\hline Richer & 0.377 & $0.17,0.81$ & 0.012 \\
\hline Richest & 0.091 & $0.02,0.35$ & 0.001 \\
\hline Poor knowledge of TB & 0.511 & $0.35,0.73$ & 0.000 \\
\hline
\end{tabular}

Chi-square $=36.568, \mathrm{df}=6, \mathrm{p}=0.000$, Nagelkerke R-square $=0.071$; Note: Factors in the equation include sex, age group, marital status, family type, educational status, occupation and distance from facility, and knowledge of TB.

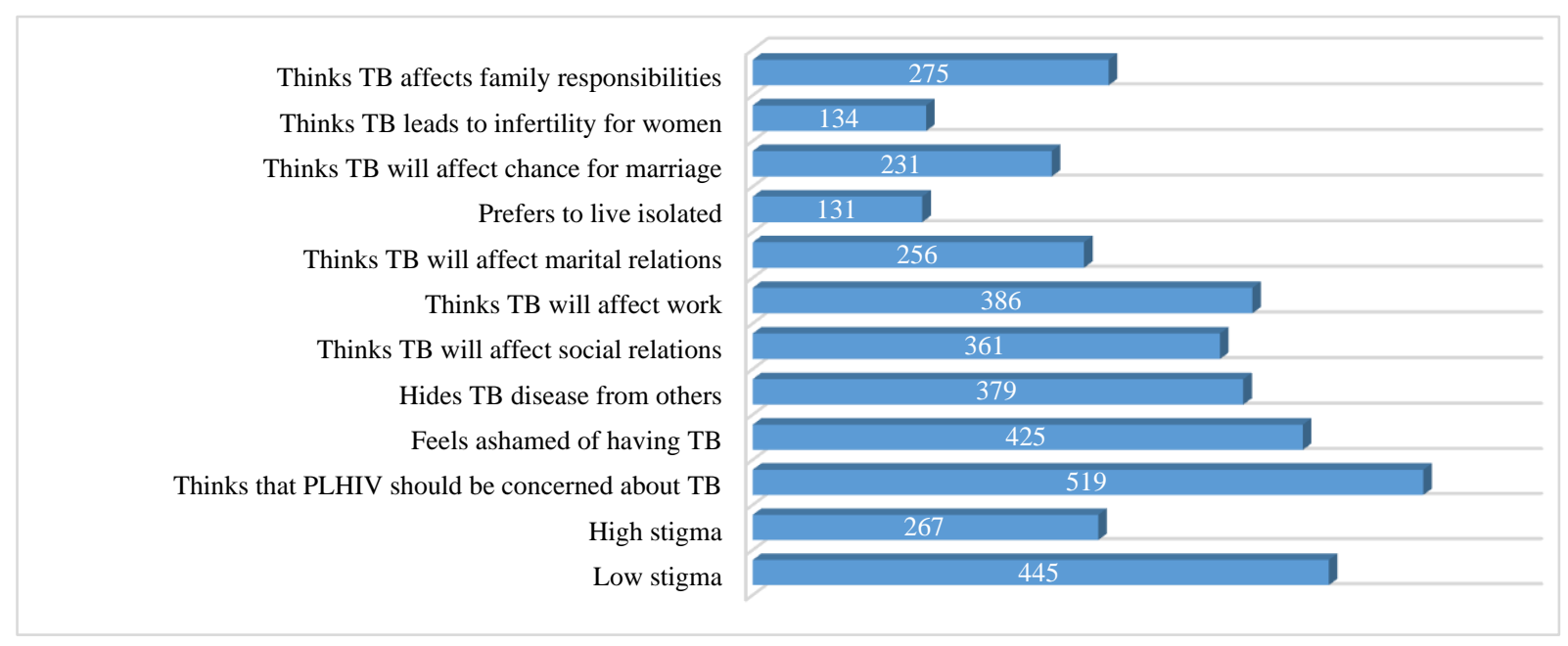

Figure 1: Patients perceived-stigma attitude and perception.

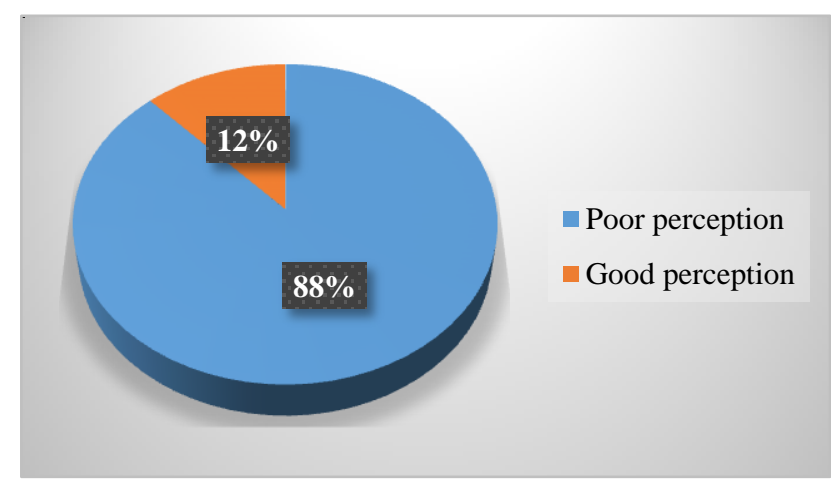

Figure 2: Patients illness perception.

\section{DISCUSSION}

We observed that the level of knowledge of patients about TB in the study was high. This finding is contrary to some studies conducted in northern Nigeria. ${ }^{13,14}$ However, studies have shown that poor knowledge of TB could contribute to high burden of TB due to patients' poor health seeking behavior such as looking for inappropriate care. ${ }^{15,16}$ Although many patients understand the need to seek care in approved health institutions, it has been shown that they do not fully assimilate the information they are receiving from the health care providers. This could be linked to lack of comprehension on TB information on the part of either the patient or nurse due to insufficient training on TB. ${ }^{17,18}$ In a setting where the knowledge of healthcare staff is limited, this may in turn limit the extent of information that is communicated, and how well the information is communicated to the patients. Ibrahim et al has shown a link between knowledge of health care workers and patients' acceptability of counselling and treatment compliance. ${ }^{19}$

The high proportion of poor perception of TB illness observed in this study is striking. This can have very adverse influence on health and recovery of patients because it can hugely influence treatment compliance.

More than a third of patients in the present study did not have good knowledge of the mode of transmission of TB. This finding correlates with other findings. ${ }^{20}$ Similar to other studies, our study found that sharing of toothbrushes, handshakes, sharing of dishes, touching of items in public places, and through sexual intercourse were reported as modes of transmission of $\mathrm{TB}^{21,22}$ Nonetheless, a high proportion of the patients noted that TB is a curable disease. The awareness that TB disease has a cure can create motivation to continue to take treatment. Another fair proportion affirmed that there is a 
vaccine for TB. The Bacillus-Calmette Guerin (BCG) is a vaccine primarily used to protect against tuberculosis among children. BCG is however, not normally given to adult except people who are at very high risk of exposure to TB. The BCG vaccine does not prevent primary infection and, more importantly, does not prevent reactivation of latent pulmonary infection, the principal source of bacillary spread in the community. ${ }^{23}$ Educating people about TB infection will enhance their knowledge or experience of the disease which will ultimately dispel myths and promote prevention habits.

The socio-demographic factors such as marital status, educational status, family type and distance to facility of patients were significant predictors for poor knowledge of TB after controlling for other factors. Age group, marital status, family type, educational status, occupation and distance from facilities. Patients who have been married before are less likely to have poor knowledge of TB compared to the never married patients after controlling for age group, family type, educational status, occupation and distance from facility. On the one hand, this finding might be attributed to the fact that married patients or those who have been married before and lost their spouses have had additional information from their partners during their interactions. On other hand, being married or in union creates the need or interest in seeking for answers to health issues and solutions.

Stigma is often recognized as an important barrier to successful care of people affected by tuberculosis. Tuberculosis has been and is still considered as a 'dirty disease', 'a death penalty' or as affecting 'guilty people'. ${ }^{24,25}$ We found that more than a third of patients experienced self-stigma in this study. Continuous patients' counselling is important in reducing selfstigmatization. ${ }^{19,26}$ It is disturbing to find that having knowledge about TB disease is not a protective factor to how patients perceive stigma. This might be because patients' awareness and deeper understanding of their illness might predispose them to negative psychological feelings like anxiety, depression and fear. It is thus highly recommended that pre-diagnostic and treatment counselling be taken very seriously in management of TB patients. This will eliminate the perception of anxiety and fear of stigma resulting from understanding of their disease. Perceived stigma was also associated with the wealth status of patients. Patients who are better off economically could easily whether off stigma.

Patients' satisfaction of quality of service is a testimonial of a more effective and efficient service delivery for TB management in the city. Majority of patients observed that TB quality of service was good. However, the perception of quality of service can be influenced by several factors.

In conclusion, the level of TB knowledge among TBinfected patients is satisfactory but perception of illness is poor. The high level of self-stigma observed in this study could create fear of being isolated and discriminated which may negatively affect treatment outcomes. The factors that predispose to poor TB knowledge are educational status, marital status and family type. Wealth status and poor knowledge are risk factors for perceived self-stigma. Programmes that are implementing intervention for TB need to be sensitive to the psychosocial environment of their patients in order to improve TB treatment outcomes.

\section{ACKNOWLEDGEMENTS}

The authors wish to thank Professor Moses Chimbari (Dean of Research, College of Health Sciences) for his support and encouragement. We also extend our profound gratitude to Dr Osman Eltayeb (WHO MDR-TB Consultant and Country Representative of Damien Foundation Belgium, Nigeria Project) and the entire team from the Oyo state Tuberculosis Control Program for their inestimable supports. Dr Oladimeji is an African Research Fellow hosted by Human Sciences Research Council (HSRC), South Africa also he also has honorary affiliation with the University of Namibia, Namibia. He is indeed grateful for the conducive research environments (HSRC and UNAM) provided for him.

Funding: Three years research postgraduate scholarship support from the College of Health Sciences and support from Damien Foundation Belgium, Nigeria project Conflict of interest: None declared

Ethical approval: The study was approved by the University of KwaZulu-Natal, South Africa Biomedical Research Ethics Committee's approval number (BE233/16)

\section{REFERENCES}

1. Word Health Organization (WHO). Tuberculosis Fact Sheet (No. 104) 2000. Available at: www who int/mediacentre/factsheets/who104/en/index.html. 2016.

2. Onyedum CC, Alobu I, Ukwaja KN. Prevalence of drug-resistant tuberculosis in Nigeria: A systematic review and meta-analysis. PloS One. 2017;12(7):e0180996.

3. Adamu AL, Gadanya MA, Abubakar IS, Jibo AM, Bello MM, Gajida AU, et al. High mortality among tuberculosis patients on treatment in Nigeria: a retrospective cohort study. BMC infectious diseases. 2017; 17(1):170.

4. World health Organization (WHO). Global tuberculosis report 2013: World Health Organization; 2013.

5. Pathmanathan I, Dokubo EK, Shiraishi RW, Agolory SG, Auld AF, Onotu D, et al. Incidence and predictors of tuberculosis among HIV-infected adults after initiation of antiretroviral therapy in Nigeria, 2004-2012. PloS one. 2017;12(3):e0173309. 
6. World Health Organization (WH0). Global tuberculosis control: a short update to the 2009 report. 2009.

7. Agho KE, Hall J, Ewald B. Determinants of the knowledge of and attitude towards tuberculosis in Nigeria. J Health, Population, Nutrition. 2014;32(3):520.

8. Kigozi G, Heunis C, Chikobvu P, Botha S, van Rensburg D. Factors influencing treatment default among tuberculosis patients in a high burden province of South Africa. Int $\mathbf{J}$ Infect Dis. 2017;54:95-102.

9. Akeju OO, Wright SC, Maja TM. Lived experience of patients on tuberculosis treatment in Tshwane, Gauteng province. Health SA Gesondheid (Online). 2017;22:259-67.

10. Alobu I, Oshi SN, Oshi DC, Ukwaja KN. Risk factors of treatment default and death among tuberculosis patients in a resource-limited setting. Asian Pacific J Trop Med. 2014;7(12):977-84.

11. Kebede EB, Sambi M. Treatment defaulter rate and associated factors among tuberculosis patients on follow up attending justh tuberculosis clinic. Journal of Infectious Diseases and Immunity. 2017;9(1):1-6.

12. Fatiregun AA, Ojo AS, Bamgboye AE. Treatment outcomes among pulmonary tuberculosis patients at treatment centres in Ibadan, Nigeria. Annals of African Med. 2009;8(2).

13. Ibrahim LM, Hadejia IS, Nguku P, Dankoli R, Waziri NE, Akhimien MO, et al. Factors associated with interruption of treatment among Pulmonary Tuberculosis patients in Plateau State, Nigeria. Pan African Med J. 2014;17(1):78.

14. Jibrin YB, Gwalabe SA, Dunga JA, Abdull MM. Treatment outcome of pulmonary tuberculosis patients in a tertiary hospital in Bauchi Northeastern Nigeria. J Med Med Sci. 2017;8(3):25-30.

15. Van Cutsem G, Isaakidis P, Farley J, Nardell E, Volchenkov G, Cox H. Infection control for drugresistant tuberculosis: early diagnosis and treatment is the key. Clin Infect Dis. 2016;62(3):238-43.

16. Edginton M, Sekatane C, Goldstein S. PatientsÕ beliefs: do they affect tuberculosis control? A study in a rural district of South Africa. Int J Tuberculosis Lung Dis. 2002;6(12):1075-82.

17. Abebe G, Deribew A, Apers L, Woldemichael K, Shiffa J, Tesfaye M, et al. Knowledge, health seeking behavior and perceived stigma towards tuberculosis among tuberculosis suspects in a rural community in southwest Ethiopia. PloS one. 2010;5(10):e13339.

18. Tobin-West CI, Isodje A. Quality and rural-urban comparison of tuberculosis care in Rivers State, Nigeria. Pan African Med J. 2016;24(1):60.

19. Ibrahim LM, Hadjia IS, Nguku P, Waziri NE, Akhimien MO, Patrobas $\mathrm{P}$, et al. Health care workers' knowledge and attitude towards TB patients under Direct Observation of Treatment in Plateau state Nigeria, 2011. Pan African Medical J. 2014;18(1):8.

20. Mulogo EM, Nahabwe C, Bagenda F, Batwala V. Determinants of treatment completion among rural smear positive pulmonary tuberculosis patients: a cross-sectional survey conducted in south-western Uganda. Infectious diseases of poverty. 2017;6(1):104.

21. World Health Organization. Global tuberculosis report 2016. 2016.

22. Kigozi NG, Heunis JC, Engelbrecht MC, van Rensburg APJ, van Rensburg HD. Tuberculosis knowledge, attitudes and practices of patients at primary health care facilities in a South African metropolitan: research towards improved health education. BMC Public Health. 2017;17(1):795.

23. Tasnim S, Rahman A, Hoque F. Patient's knowledge and attitude towards tuberculosis in an urban setting. Pulmonary Med. 2012;2012.

24. Wandwalo E, Mørkve O. Knowledge of disease and treatment among tuberculosis patients in Mwanza, Tanzania. Int $\mathbf{J}$ Tuberculosis Lung Dis. 2000;4(11):1041-6.

25. Wang J, Fei Y, Shen H, Xu B. Gender difference in knowledge of tuberculosis and associated healthcare seeking behaviors: a cross-sectional study in a rural area of China. BMC Public Health. 2008;8(1):354.

26. Hoa NP, Thorson AE, Long NH, Diwan VK. Knowledge of tuberculosis and associated healthseeking behaviour among rural Vietnamese adults with a cough for at least three weeks. Scandinavian J Public Health. 2003;31(62):59-65.

Cite this article as: Oladimeji $\mathrm{O}$, Tsoka-Gwegweni JM, Adeyinka DA, Makola L, Mitonga KH, Udoh EE, et al. Knowledge, attitude and perception of tuberculosis management among tuberculosisinfected patients in resource constraint setting: field experience from Oyo state, South-West, Nigeria. Int J Community Med Public Health 2018;5:1694-706. 